\title{
Acquired resistance to DZNep-mediated apoptosis is associated with copy number gains of AHCY in a B-cell lymphoma model
}

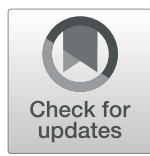

Chidimma Agatha Akpa ${ }^{1,2^{*}}$, Karsten Kleo ${ }^{1}$, Elisabeth Oker ${ }^{1}$, Nancy Tomaszewski ${ }^{1}$, Clemens Messerschmidt ${ }^{3}$, Cristina López ${ }^{4}$, Rabea Wagener ${ }^{4}$, Kathrin Oehl-Huber ${ }^{4}$, Katja Dettmer $^{5}$, Anne Schoeler $^{6,7}$, Dido Lenze ${ }^{1}$, Peter J. Oefner ${ }^{2}$, Dieter Beule ${ }^{3}$, Reiner Siebert ${ }^{4}$, David Capper $^{2,6,7}$, Lora Dimitrova ${ }^{1}$ and Michael Hummel ${ }^{1,2}$

\begin{abstract}
Background: Enhancer of zeste homolog 2 (EZH2) is considered an important driver of tumor development and progression by its histone modifying capabilities. Inhibition of EZH2 activity is thought to be a potent treatment option for eligible cancer patients with an aberrant EZH2 expression profile, thus the indirect EZH2 inhibitor 3Deazaneplanocin A (DZNep) is currently under evaluation for its clinical utility. Although DZNep blocks proliferation and induces apoptosis in different tumor types including lymphomas, acquired resistance to DZNep may limit its clinical application.

Methods: To investigate possible mechanisms of acquired DZNep resistance in B-cell lymphomas, we generated a DZNep-resistant clone from a previously DZNep-sensitive B-cell lymphoma cell line by long-term treatment with increasing concentrations of DZNep (ranging from 200 to $2000 \mathrm{nM}$ ) and compared the molecular profiles of resistant and wild-type clones. This comparison was done using molecular techniques such as flow cytometry, copy number variation assay (OncoScan and TaqMan assays), fluorescence in situ hybridization, Western blot, immunohistochemistry and metabolomics analysis.

Results: Whole exome sequencing did not indicate the acquisition of biologically meaningful single nucleotide variants. Analysis of copy number alterations, however, demonstrated among other acquired imbalances an amplification (about 30 times) of the S-adenosyl-L-homocysteine hydrolase (AHCY) gene in the resistant clone. AHCY is a direct target of DZNep and is critically involved in the biological methylation process, where it catalyzes the reversible hydrolysis of S-adenosyl-L-homocysteine to L-homocysteine and adenosine. The amplification of the AHCY gene is paralleled by strong overexpression of AHCY at both the transcriptional and protein level, and persists upon culturing the resistant clone in a DZNep-free medium.

(Continued on next page)
\end{abstract}

\footnotetext{
* Correspondence: chidimma.akpa@charite.de

'Department of Experimental Hematopathology, Institute of Pathology, Charité Medical University, Berlin, Charitéplatz 1, 10117 Berlin, Germany

${ }^{2}$ Berlin School of Integrative Oncology, Charité - Medical University of Berlin, Berlin, Germany

Full list of author information is available at the end of the article
}

(C) The Author(s). 2020 Open Access This article is licensed under a Creative Commons Attribution 4.0 International License, which permits use, sharing, adaptation, distribution and reproduction in any medium or format, as long as you give appropriate credit to the original author(s) and the source, provide a link to the Creative Commons licence, and indicate if changes were made. The images or other third party material in this article are included in the article's Creative Commons licence, unless indicated otherwise in a credit line to the material. If material is not included in the article's Creative Commons licence and your intended use is not permitted by statutory regulation or exceeds the permitted use, you will need to obtain permission directly from the copyright holder. To view a copy of this licence, visit http://creativecommons.org/licenses/by/4.0/. The Creative Commons Public Domain Dedication waiver (http://creativecommons.org/publicdomain/zero/1.0/) applies to the data made available in this article, unless otherwise stated in a credit line to the data. 
(Continued from previous page)

Conclusions: This study reveals one possible molecular mechanism how B-cell lymphomas can acquire resistance to DZNep, and proposes AHCY as a potential biomarker for investigation during the administration of EZH2-targeted therapy with DZNep.

Keywords: 3- Deazaneplanocin a (DZNep), B-cell lymphoma, Enhancer of zeste homolog 2 (EZH2), S-adenosyl-Lhomocysteine hydrolase (AHCY)

\section{Background}

The development of drug resistance to cancer chemotherapeutics remains a major concern in most treatment regimens. Several epigenetic-based therapies are under investigation or being employed for treatment of patients with lymphomas of B-cell origin. This is due to the important role that epigenetic alterations play in promoting tumor development and progression via downregulation of tumor suppressor genes [1]. These epigenetic modifications may involve covalent post-translational modifications at the N-termini of histones or changes in the methylation pattern of cytosine bases within the DNA, especially at CpG sites [2]. Histones are important structural components of the cell that package and organize DNA into nucleosomal units and various posttranslational modifications of histones are known to contribute to transcriptional gene activity in conjunction with other mechanisms [3-8].

Enhancer of zeste homolog 2 (EZH2) is a histone methyltransferase that is involved in cellular differentiation and development in both health and disease. EZH2 promotes transcriptional repression by catalyzing the trimethylation of lysine 27 on histone 3 (H3K27me3) - a repressive histone mark. In lymphoma and other malignancies, EZH2 gain-offunction mutations and overexpression are considered important drivers of oncogenesis because of their role in silencing tumor suppressor genes regulating apoptosis, cell cycle regulation, proliferation, migration and differentiation [9-14]. Due to its oncogenic role, the targeting of EZH2 might be a promising approach for lymphoma therapy. 3Deazaneplanocin A (DZNep) is an indirect inhibitor of EZH2 currently in the pre-clinical phase of drug development and has been shown to promote apoptosis in various primary tumor cells and cancer cell lines [15-20]. The apoptotic effects mediated by DZNep application are more pronounced in cancer cells, with minimal effects on normal cells, and are fostered by the inhibition of the repressive H3K27me3 mark [15, 18, 21].

DZNep directly inhibits the enzyme S-adenosyl-Lhomocysteine hydrolase (AHCY) that catalyzes the reversible hydrolysis of S-adenosyl-L-homocysteine (SAH) to L-homocysteine and adenosine. The direct inhibition of AHCY by DZNep leads to the build-up of the substrate SAH, which in turn causes a negative feedback inhibition of methyltransferases such as EZH2 [22]. Proper functioning of AHCY is essential for the efficient maintenance of histone methylation levels in the cell [23]. Alterations in AHCY function have been linked to cancer with varying outcomes depending on the cancer entity involved. For example, with lowered AHCY activity, the invasiveness of breast cancer and glioblastoma cell lines decreases [24, 25]. Furthermore, in hepatocellular carcinoma cells, reduced AHCY activity is associated with cell cycle inhibition and a lowered proliferation rate [23]. In esophageal squamous cell carcinoma, however, elevated AHCY levels had no effect on cell proliferation but promoted apoptosis and inhibited cell migration and adhesion [26]. Besides, aberrant AHCY expression has been observed with the transformation of follicular lymphoma to diffuse large B-cell lymphoma [27].

In this study, we investigated the underlying molecular mechanism of resistance of a B-cell lymphoma model to DZNep using a DZNep-resistant clone generated from a DZNep-sensitive cell line. We identified AHCY as a potential biomarker that could be of predictive relevance for therapeutic inhibition of EZH2 using DZNep.

\section{Methods}

\section{Drug, cell lines and culture conditions}

DZNep (Selleckchem, Germany) was dissolved in sterile water following the manufacturer's recommendation as previously described [20].

The sporadic Burkitt lymphoma cell line BLUE-1 (ACC-594, from German Collection of Microorganisms and Cell Cultures (DSMZ) Germany) was cultured in RPMI 1640 (ThermoFisher Scientific, Germany) medium enriched with $20 \%$ fetal calf serum (PAN-Biotech, Germany). Cell lines were tested and confirmed mycoplasma negative with the MycoAlert Mycoplasma Detection kit (Lonza, Germany). All cell lines were incubated at $37^{\circ} \mathrm{C}$ at $5 \% \mathrm{CO}_{2}$.

Generation of a DZNep resistant clone was achieved by splitting the BLUE-1 culture into a control group and a treatment group (Fig. 1a). The treated group received increasing concentrations of DZNep starting from 200 $\mathrm{nM}$ up to $2000 \mathrm{nM}$ over a period of 7 months. The cells were split 3 times a week and fresh medium without or with DZNep was added to the control and treated cells, respectively. Vital cells were counted each time by flow cytometry before the cells were split. Cryostocks were 


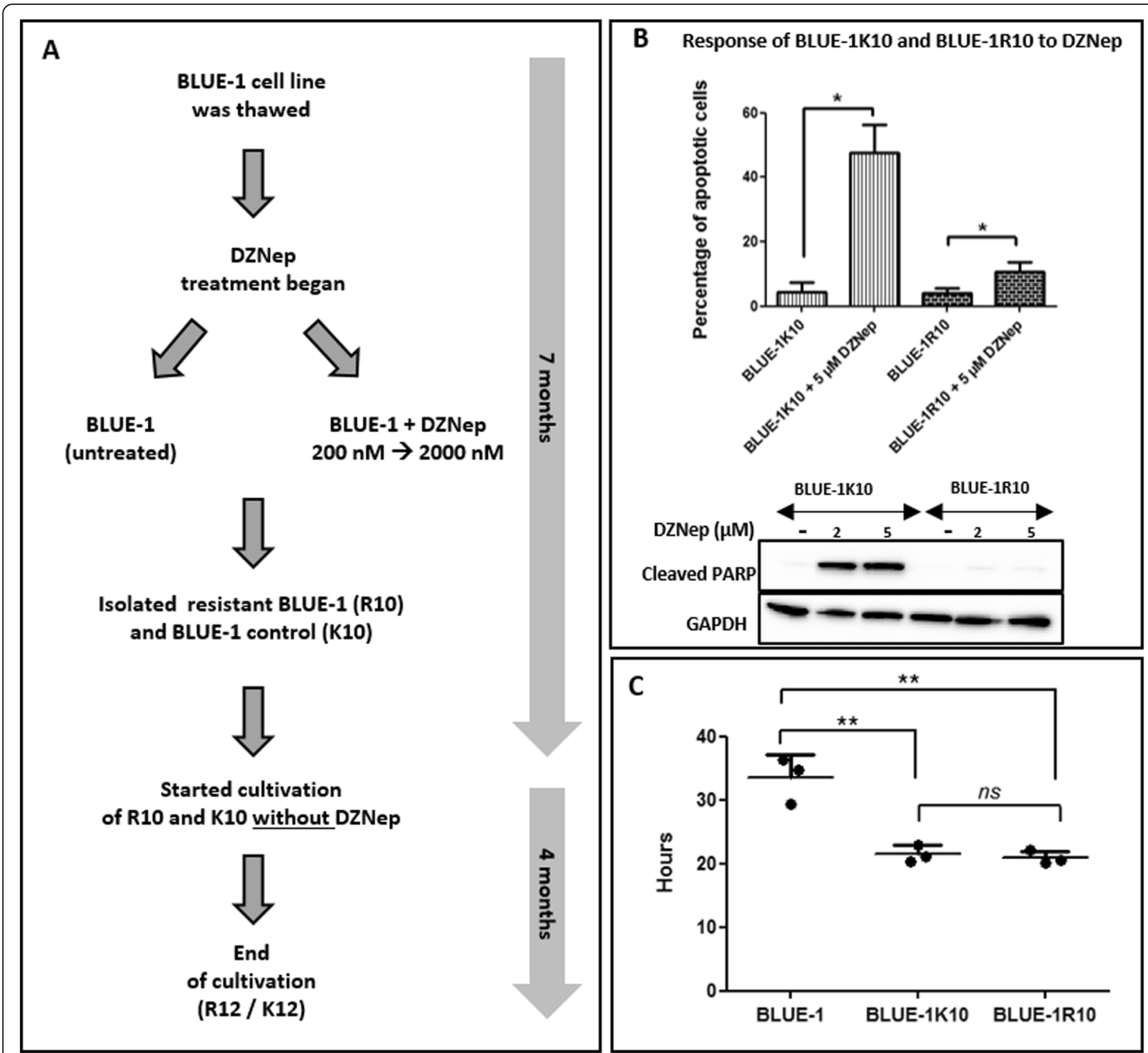

Fig. 1 Generation and characterization of a DZNep-resistant clone. a Scheme of the generation of the DZNep-resistant clone and its control. b Comparison of the apoptotic response of BLUE-1 K10 (control) and BLUE-1R10 to DZNep. Above: The cell lines BLUE-1R10 and BLUE-1 K10 were either treated with $5 \mu \mathrm{M}$ DZNep or untreated for $72 \mathrm{~h}$. Cells were harvested and the percentage of apoptotic cells was determined by flow cytometry. Data is shown as mean plus standard deviation (SD) of three biological replicates. Below: Western blot analysis was performed using total protein lysates from both cell lines either untreated or treated with $2 \mu \mathrm{M}$ and $5 \mu \mathrm{M}$ DZNep, respectively. GAPDH was used as a loading control for the Western blot. The full-length blot is presented in Additional file 6: Fig. S5. The FUSION-CAP Software was used for Western blot image analysis. c Comparison of the doubling time of BLUE-1, BLUE-1 K10 and BLUE-1R10. The three cell lines were cultivated at a seeding density of $2 \times 10^{5}$ cells in 6 -well culture plates. The number of vital cells were measured at $24 \mathrm{~h}, 48 \mathrm{~h}$ and $72 \mathrm{~h}$ by flow cytometry. Doubling time is shown as mean plus SD of triplicate measurements. ns: not significant

made every third to fourth week from both cell cultures. At 7 months, DZNep pressure on cultures of the treated group was removed by growing both untreated (BLUE-1 K10) and treated (BLUE-1R10) cells in medium without DZNep. About 4 months later, both cell cultures were harvested (BLUE-1 K12 and BLUE-1R12) and frozen. Prior to further use, frozen cells were thawed and maintained in a DZNep-free medium for at least 1 week.

\section{Flow cytometry}

The BD Accuri C6 flow cytometer (Becton Dickinson Biosciences, USA) was used for the measurement of apoptotic cells and determination of the doubling time of cells. Measurement of apoptosis was performed after staining $3 \times 10^{5}$ cells from the cell cultures with a mixture of Annexin V (Biolegend, USA) and propidium iodide (Biolegend, USA). Doubling time was determined by 
seeding the cells in 6 -well plates at a cell density of $2 \times$ $10^{\wedge} 5$ cells $/ \mathrm{ml}$. The number of vital cells were counted after $24 \mathrm{~h}, 48 \mathrm{~h}$ and $72 \mathrm{~h}$ by flow cytometry. The doubling time during the exponential growth phase was subsequently calculated using the formula DT $=\mathrm{T} \ln 2 / \ln (\mathrm{Xe} /$ $\mathrm{Xb}$ ) (https://www.atcc.org/ /media/PDFs/Culture\%2 0Guides/AnimCellCulture_Guide.ashx), where DT represents the doubling time (in hours), $\mathrm{T}$ symbolizes the incubation time (in hours), Xe indicates the cell number at the end of the incubation time, and $\mathrm{Xb}$ is the cell number at the beginning of the incubation time.

\section{Western blotting, RNA isolation and real-time reverse transcriptase polymerase chain reaction (RT-PCR)}

Cell lysis and Western blot were carried out as described previously [20]. Twelve percent Expedeon RunBlue SDS protein gels (Biozol Diagnostica Vertrieb $\mathrm{GmbH}$, Germany) were utilized for the run. The primary and secondary antibodies used for Western blot are shown in Table 1.

RNA was isolated using the RNeasy Midi Kit (Qiagen, Germany) adhering to the manufacturer's recommendations and reverse transcribed to cDNA using TaqMan Reverse Transcription reagents (ThermoFisher Scientific, Germany) on a T3 thermocycler (Biometra $\mathrm{GmbH}$, Germany). Real-time RT-PCR was carried out using TaqMan Gene Expression Assays and TaqMan Gene Expression Master Mix (ThermoFisher Scientific, Germany) following the manufacturer's protocol on the StepOnePlus Real-Time PCR System (ThermoFisher Scientific, Germany). Beta-2 microglobulin (B2M) and succinate dehydrogenase (SDHA) genes were used as endogenous controls and the $\Delta \Delta C_{t}$ method [28] was followed for relative mRNA quantification. Details of the respective TaqMan assays used are listed in Table 2.

DNA isolation, whole exome sequencing (WES), copy number variation (CNV) assay and OncoScan CNV assay Genomic DNA was isolated from the cell lines using QIAamp DNA Mini Kit (Qiagen, Germany), following
Table 2 TaqMan assays used for gene expression and copy number variation (CNV) analysis

\begin{tabular}{lllll}
\hline \multicolumn{2}{l}{ TaqMan Assays } & & & \\
\hline & Targets & Assay ID & Type & Company \\
\hline $\begin{array}{l}\text { CDNA } \\
\text { target }\end{array}$ & AHCY & Hs00898137_g1 & $\begin{array}{l}\text { Gene expression } \\
\text { assay }\end{array}$ & $\begin{array}{l}\text { ThermoFisher } \\
\text { Scientific }\end{array}$ \\
& B2M & Hs00984230_m1 & $\begin{array}{l}\text { Gene expression } \\
\text { assay }\end{array}$ & $\begin{array}{l}\text { ThermoFisher } \\
\text { Scientific }\end{array}$ \\
& SDHA & Hs00417200_m1 & $\begin{array}{l}\text { Gene expression } \\
\text { assay } \\
\text { CNV assay }\end{array}$ & $\begin{array}{l}\text { ThermoFisher } \\
\text { Scientific }\end{array}$ \\
$\begin{array}{llll}\text { TDNA } \\
\text { target }\end{array}$ & AHCY & Hs02422126_cn & Scientific \\
\hline
\end{tabular}

the manufacturer's instructions. WES was performed on genomic DNA at the Berlin Institute of Health core facility Genomics, Berlin, Germany. Sequencing libraries were prepared with the SureSelect ${ }^{\mathrm{XT}}$ Human All Exon v4 library kit (Agilent, Germany) following the manufacturer's instructions. Cluster Generation was done with the aid of TruSeq PE Cluster Kit v4 (Illumina, USA) and the resulting templates sequenced on an Illumina HiSeq2000 sequencer (at least 150 million reads with a sequencing depth of greater than 160x) using the Illumina HiSeq SBS 250 cycle kit v4.

Data analysis was performed using BWA-MEM [29] to map each whole-exome data set against the reference genome GRCh37. Samblaster [30] was used to mark duplicates. To detect copy number changes, DNA profiles from the respective cell lines were compared (BLUE1R10 against BLUE-1 K10) with CNVkit [31]. The copy number changes were prioritized according to their log2 fold-change and custom plots of amplified regions created using CNVkit plotting functions.

$\mathrm{CNV}$ analysis was performed on genomic DNA from the respective BLUE-1 cell lines, controls and patient samples by applying the TaqMan copy number assay (assay ID: Hs02422126_cn) to the AHCY gene on chromosome 20. The assay covers intron 7 and exon 8 on the reference genome GRCh37 and was performed according to the manufacturer's recommendations. Data

Table 1 List of antibodies

\begin{tabular}{|c|c|c|c|c|}
\hline \multirow[t]{3}{*}{ Target Protein } & \multicolumn{4}{|c|}{ Primary antibody } \\
\hline & \multicolumn{2}{|l|}{ Application } & \multirow[t]{2}{*}{ Company } & \multirow[t]{2}{*}{ Clone name / catalogue number } \\
\hline & Western blot & Immunohistochemistry & & \\
\hline GAPDH & $\checkmark$ & & Cell Signaling Technology & $14 C 10$ \\
\hline Histone 3 & $\checkmark$ & & Cell Signaling Technology & $96 C 10$ \\
\hline Cleaved PARP & $\checkmark$ & & Cell Signaling Technology & Asp214 \\
\hline $\mathrm{AHCY}$ & $\checkmark$ & $\checkmark$ & OriGene & TA332593 \\
\hline \multicolumn{5}{|l|}{ Secondary antibody } \\
\hline Anti-rabbit HRP-conjugated & $\checkmark$ & & GE Healthcare & NA934V \\
\hline Anti-mouse HRP-conjugated & $\checkmark$ & & Agilent & P0447 \\
\hline
\end{tabular}


analysis was done using the CopyCaller software version 2.1 (ThermoFisher Scientific, Germany).

OncoScan CNV assay Kit was also used to perform copy number analysis according to standard protocols (Affymetrix) [32]. The Chromosome Analysis Suite 4.0 (ChAS) (ThermoFisher, Germany) software was used to visualize, analyze and summarize the chromosomal aberrations, including gains, losses, and loss of heterozygosity (LOH). The non-FFPE analysis work-flow was applied. Criteria for copy number alterations include chromosomal changes encircling at least 20 informative probes, with a minimum size event of at least $100 \mathrm{~kb}$, with median $\log 2$ Ratio $+/-0.3$, and showing a CNN-LOH more than $5 \mathrm{Mb}$. Individual copy number analysis for each BLUE-1 cell line, as well as, comparative analysis between the three cell lines (BLUE-1, BLUE-1 K10, and BLUE-1R10) was done. In addition, we manually inspected all the aberrations filtered out due to the criteria described above and included only those aberrations showing differences in the B-allele frequency (BAF).

\section{Clonality studies}

B-cell clonality studies were performed to determine the immunoglobulin heavy chain (IGH) rearrangements for BLUE-1, BLUE-1 K10 and BLUE-1R10 using a multiplex PCR method developed within the EuroClonality/ BIOMED-2 collaborative study, BMH4-CT98-3936 [33] for all three IGH frame work regions. After PCR on the ProFlex PCR Thermocycler (ThermoFisher Scientific, Germany), gel electrophoresis was performed to check the amplification of PCR products. For single base pair resolution, GeneScan analysis (capillary electrophoresis) of the IGH PCR products was performed with the 3500 series Genetic Analyser (ThermoFisher Scientific, Germany). The sizes of the various PCR products were determined using the GeneMapper 4.0 software (ThermoFisher Scientific, Germany).

\section{Immunohistochemistry and fluorescence in situ hybridization (FISH) analysis}

Immunohistochemistry (IHC) was performed using sections of formalin-fixed paraffin-embedded (FFPE) cell line blocks as described [34]. The primary antibody used in this case was anti-AHCY antibody (Table 1). FISH was performed on sections of formalin-fixed paraffinembedded (FFPE) cell line blocks as described [35, 36]. This was carried out using orange-labeled AHCY genespecific probes (product name: AHCY-20-OR) and green-labeled chromosome 20-control (centromeric) probes (product name: CHR20-10-GR) (both purchased from Empire Genomics, USA). DakoCytomation Hybridizer (Dako/Agilent, Germany) was used for FISH probe hybridization, while nuclear counterstaining was done with the aid of Dako fluorescence mounting medium containing DAPI (Dako/Agilent, Germany). Visualization and analysis (of at least 50 intact nuclei) were performed with the Zeiss Axio Imager Z1 (Zeiss, Germany) and the Isis imaging software version 5.3.1 (Metasystems, Germany).

\section{Cytogenetics, metabolomics and global DNA methylation analysis}

Profiling of short tandem repeats (STR) for authentication of the cell lines BLUE-1, BLUE-1 K10 and BLUE1R10 using the StemElite kit (Promega) was performed as previously described [32]. Conventional cytogenetic analysis was performed as reported [37] and the karyotypes were described according to ISCN guidelines (2013).

The intermediates of methionine and polyamine metabolism in BLUE-1, BLUE-1 K12 and BLUE-1R12 cell extracts were measured by liquid chromatographytandem mass spectrometry following an established protocol [38]. Genome-wide DNA methylation analysis was done on BLUE-1, BLUE-1 K10 and BLUE-1R10 using the Infinium MethylationEPIC BeadChip (Illumina, USA) as described [39]. Copy number plots were generated from the raw output data (.idat files) using the 'conumee' $R$ package in Bioconductor [39, 40].

\section{Statistical analysis}

Statistical analysis was done using the GraphPad Prism 5 software (GraphPad Software, California, USA). Statistical significance was evaluated using the Mann-Whitney $\mathrm{U}$ test (two-tailed) for pairwise comparisons, and oneway ANOVA with the Tukey post-hoc test for group comparisons. $p$ values less than 0.05 were considered significant.

\section{Results}

Generation and characterization of a DZNep-resistant cell line clone

We generated a DZNep-resistant clone by subjecting the DZNep-sensitive Burkitt lymphoma cell line BLUE-1 [20] to progressively increasing concentrations of DZNep for up to 7 months (Fig. 1a and Methods section). Analyses were performed with the resistant BLUE1 subclones (BLUE-1R10 or BLUE-1R12) and their respective controls. To analyze the response of the generated DZNep-resistant clone to DZNep, we treated the clone and its corresponding control with $5 \mu \mathrm{M}$ DZNep for $72 \mathrm{~h}$ and then measured the percentage of apoptotic cells. The control cell line, BLUE-1 K10, exhibited strong apoptosis with about $50 \%$ apoptotic cells in comparison to the resistant BLUE-1R10 clone, which displayed only about $10 \%$ apoptotic cells following treatment with DZNep (Fig. 1b). Furthermore, Western blot analysis 
performed on total protein lysate obtained after treatment of both cell lines with $2 \mu \mathrm{M}$ and $5 \mu \mathrm{M}$ DZNep revealed an increase in the expression of cleaved PARP, indicating apoptosis in the DZNep-treated cells of BLUE-1 K10 in relation to BLUE-1R10 (Fig. 1b). The doubling time of the DZNep-resistant clone BLUE-1R10 was also compared with that of the corresponding control BLUE-1 K10 and the parent cell line BLUE-1. This revealed that both BLUE-1R10 and BLUE-1 K10 had shorter doubling times than BLUE-1 (Fig. 1c).

To determine the identity of the generated clone, we explored the STR profile and determined the clonality of BLUE-1R10. The results were then compared with those of its corresponding control BLUE-1 K10 and the parent cell line BLUE-1. The STR profile analysis for the three cell lines when compared with the DSMZ STR profiling database revealed an STR profile of a BLUE-1 cell line, confirming their authenticity. Furthermore, the IGH chain gene rearrangement patterns for the three cell lines were identical (Additional file 1: Figure S1). We also performed genomic characterization of the cell lines using conventional cytogenetics and copy number analysis by OncoScan CNV assay. Only minor differences in the karyotype were observed upon analysis of the three cell lines (Additional file 2: Figure S2). Upon further examination the copy number data, we detected genomic aberrations exclusive to each of the three BLUE-1 cell lines (Additional file 5: Table S1). We detected three abnormalities only present in BLUE-1R10 as compared to the parental BLUE-1 and BLUE-1 K10 cell lines. These include one loss in 4q12q12, one high copy gain in $6 \mathrm{q} 14.3 \mathrm{q} 14.3$, and one $\mathrm{LOH}$ in 20p12.2p13. Moreover, we observed aberrations solely in the parental BLUE-1 cell line, including gains in $6 \mathrm{p} 22.1 \mathrm{p} 25.3,19 \mathrm{p} 13.11 \mathrm{p} 13.3$, and 19p13.11q13.43, which may have been lost due to prolonged culture conditions. We also distinguished common copy number aberrations in BLUE-1 and BLUE-1R10 cell lines, but not shown in the BLUE-1 K10 (Additional file 5: Table S1).

\section{Identification of biomarkers for resistance to DZNep}

To probe the underlying mechanism of resistance of BLUE-1R10 to DZNep, we performed WES of genomic DNA obtained from BLUE-1R10 and its corresponding control BLUE-1 K10. Additional copy number variant analysis was performed on the WES data using the software tool CNVkit. This analysis identified a small focal, approximately 30 -fold copy number gain in the region spanning the $A H C Y$ gene and the proximal part of the ITCH gene on chromosome 20 (Fig. 2a). Upon manual inspection of this specific genomic region in the OncoScan data, we confirmed the presence of this high copy number gain including the $A H C Y$ gene (Additional file 3: Figure S3). It was not called in the initial analysis due to applied detection criteria for the analysis which filtered the amplicon out due to its small genomic size and low marker content (see material and method sections for more details).

We validated this $A H C Y$ gain on the DNA level using two methods. First, we used the TaqMan Copy Number Assay to analyze DNA obtained from cryostocks collected at the various time-points during the generation of the resistant clone, including BLUE-1 as the reference cell line. We noted a clear copy number gain in $A H C Y$ beginning with BLUE-1R6 (preserved after almost 5 months of treatment with DZNep) and increasing thereafter (Fig. 2b). The $A H C Y$ copy number gain was further confirmed by a CNV analysis using global DNAmethylation data from BLUE-1R10, and comparing it with data from BLUE-1 K10 and BLUE-1. Here, the gain in $A H C Y$ copy number on chromosome 20 was also obvious in the resistant clone in comparison to the respective control and the parent cell line (Fig. 2c).

\section{AHCY copy number gain at the chromosomal and transcriptional level}

On the chromosomal level, we confirmed $A H C Y$ amplification in BLUE-1R10 and BLUE-1R12, by performing a FISH study using target-specific probes for $A H C Y$ on chromosome 20q11.22. Our FISH data (Fig. 3a) revealed cluster-type amplification and large $A H C Y$-gene clusters in form of dense clouds of labeled regions suggestive for hsr-regions in cells of BLUE-1R10, when compared with its control BLUE-1 K10 and BLUE-1.

To ascertain the expression of AHCY, we performed a real-time PCR using the TaqMan gene expression assay on cDNA from the resistant clone, the respective controls and the parent cell line BLUE-1. The results display overexpression of AHCY in the resistant clone BLUE$1 \mathrm{R} 10$ and a further increase in BLUE-1R12 (Fig. 3b).

\section{$A H C Y$ gain at the protein level and metabolomics studies} $\mathrm{AHCY}$ expression at the protein level was determined using two different methods, IHC and Western blot. The IHC results show increased AHCY expression in the resistant clone in comparison to its control and the parent cell line (Fig. 4a). The Western blot result (Fig. 4b) confirmed that AHCY is overexpressed in BLUE-1R10, with a higher expression in BLUE-1R12. The respective controls and parent BLUE-1 cell line had a similar level of AHCY expressed.

To understand the dynamics of the concentrations of methionine intermediates in the resistant clone, its control and the parent cell line, we employed a high performance liquid chromatography-tandem mass spectrometry method. We notice a similar distribution pattern for S-adenosyl-L-homocysteine (SAH), adenine and adenosine in BLUE-1 and the control cell line 


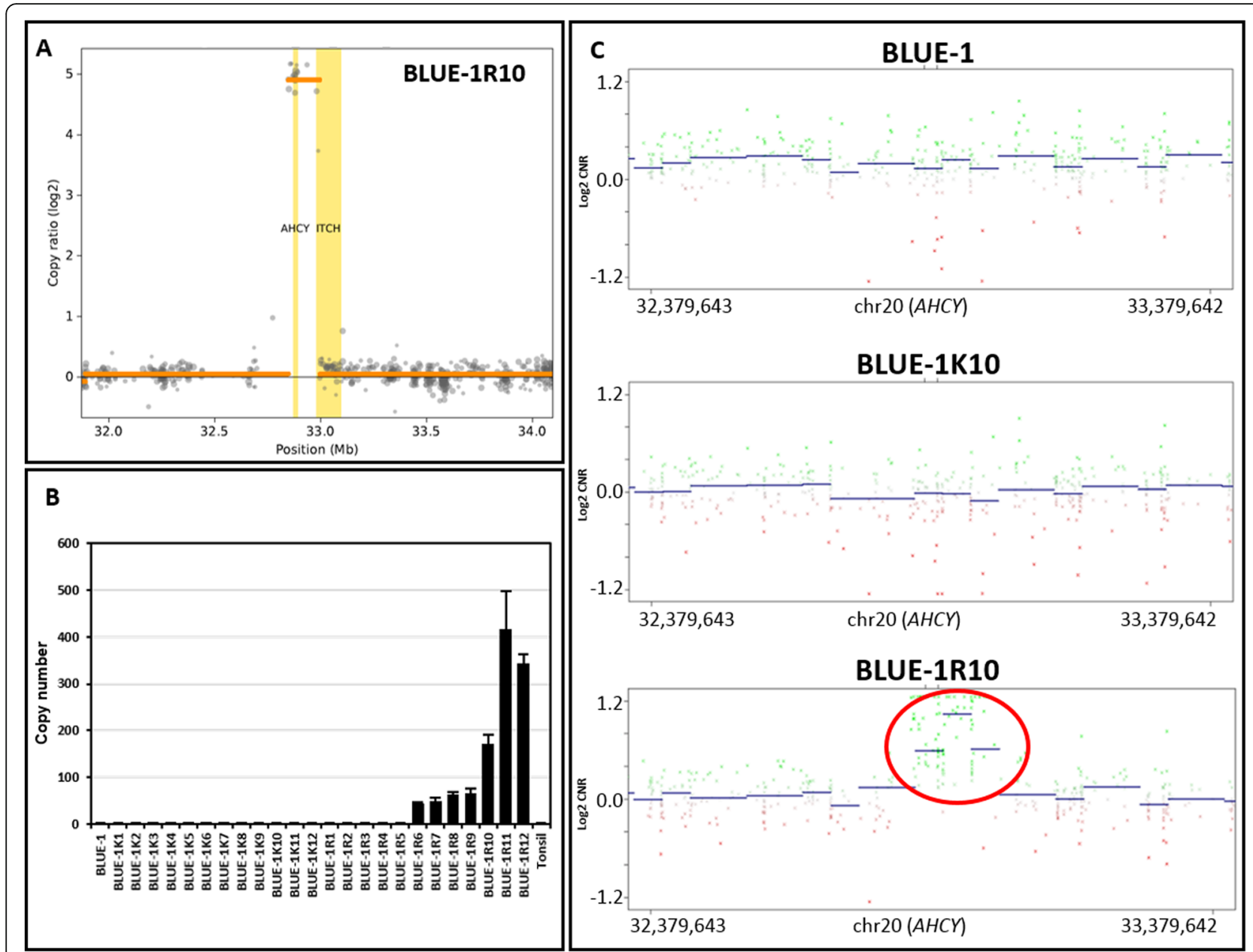

Fig. 2 AHCY copy number gain in the DZNep-resistant clone. a Log2 copy ratio plot of copy number variation regions in BLUE-1R10 in relation to BLUE-1 K10. The gray dots represent copy ratio values across different bins, the orange line shows segments and the yellow vertical lines indicate the respective genes. $\mathbf{b}$ Evolution of AHCY copy number in the resistant clone. Genomic DNA from the cell lines was subjected to the TaqMan copy number assay (ID: Hs02422126_cn). The real-time PCR read-out and copy number was analyzed with the CopyCaller software. A human tonsil DNA sample was used as a calibrator for the TaqMan copy number assay. c CNV plots calculated from global DNA methylation array data. Chromosome 20 locus on BLUE-1, BLUE-K10 and BLUE-1R10 was analyzed for variations in AHCY copy number. The $y$-axis represent the log2 copy number ratio (CNR). Amplifications represent positive deviations from the baseline while losses indicate negative deviations from the baseline (0.0). Encircled in red, shows AHCY copy number gain in BLUE-1R10

BLUE-1 K12. The resistant clone (BLUE-1R12), however, displayed a significant increase $(p<0.05)$ in the level of both adenine and adenosine in comparison to its control BLUE-1 K12 (Fig. 4c).

\section{$A H C Y$ copy number gain in primary lymphoma samples}

We examined the frequency of $A H C Y$ copy number gains in a small series of B-cell lymphomas by examining 12 primary lymphoma samples consisting of Burkitt lymphoma, diffuse large B-cell lymphoma, follicular lymphoma, primary mediastinal B-cell lymphoma and anaplastic large cell lymphoma. We performed the TaqMan CNV assay using genomic DNA isolated from these samples. Subsequent analysis of the $A H C Y$ copy number with the CopyCaller software revealed a predicted $A H C Y$ copy number of 2 for all but one sample, which had a predicted copy number of 1 (Additional file 4: Figure S4). In addition, we checked the frequency of $A H C Y$ copy number alterations in B-cell lymphoma by analyzing published genomic data from 1295 B-cell lymphoma samples curated from 5 different studies on the cBio Cancer Genomics Portal $[41,42]$ but no $A H C Y$ genomic alterations were detected.

\section{Discussion}

Acquired resistance to small molecule inhibitors used in cancer treatment remains a huge problem in cancer therapy. Although many cancer types respond to initial therapy, there is the uncertainty of resistance arising against the utilized drug [43]. Various molecular 

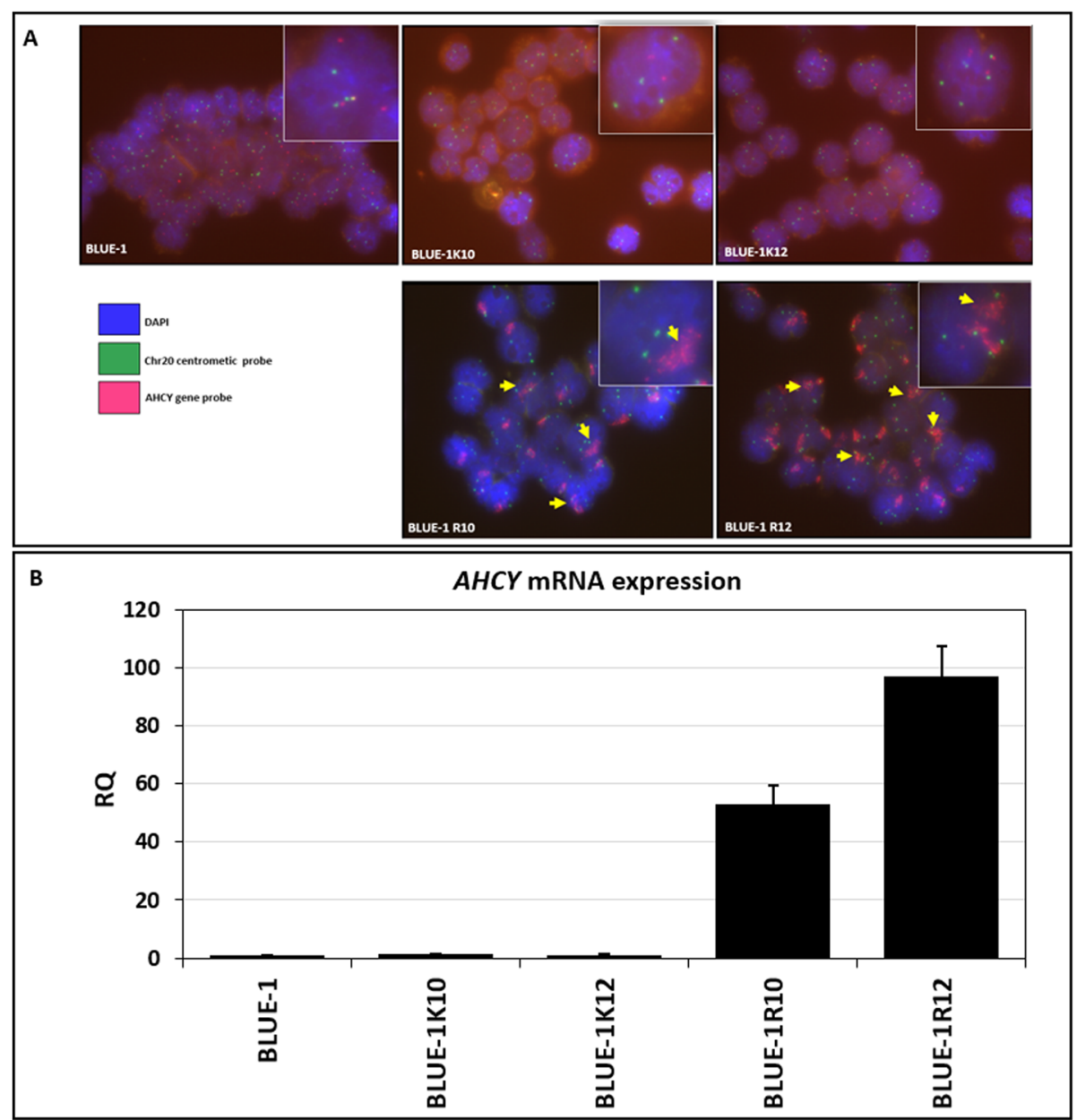

Fig. 3 AHCY copy number gain in the DZNep-resistant clone: chromosomal and transcriptional validation. a FISH analysis using AHCY targetspecific probes in the resistant clone and its control. Yellow arrows show in pink color a cluster-type amplification of AHCY. Green dots represent the centromere of chromosome 20. b Transcriptional expression of AHCY. cDNA was synthesized from the RNA of all cell lines. Relative gene expression (shown on the $y$-axis) was quantified using the AHCY gene expression assay, with B2M and SDHA used as an endogenous control. RQ is shown as mean plus SD of triplicate measurements

mechanisms are involved in the resistance of tumor cells to therapy [44-46]. DZNep - an indirect EZH2 inhibitor - is known to be efficacious against many different types of cancer and, hence, may make its way into clinical trials. Already, genetic determinants of sensitivity to DZNep-mediated apoptosis have been described for gastric cancer and multiple myeloma tumor cells respectively $[47,48]$. In this study, we aimed to investigate the molecular mechanism underlying acquired resistance to DZNep in B-cell lymphoma, and to identify biomarkers predictive of the therapeutic success of EZH2 inhibition with DZNep. To achieve this, we generated and investigated a DZNep-resistant clone. The continued resistance of the clone (BLUE-1R10) to DZNep following treatment with DZNep as well as upon cultivation in a DZNep-free medium implies that a permanent change has occurred on the genomic level in this clone. We analyzed the proliferation rate of BLUE-1R10, comparing its doubling time with that of BLUE-1 K10 and BLUE-1. The shorter doubling time observed in the resistant clone and its control in relation to the parent cell line is in contrast to a previous report of an increased doubling time in two colon cancer cell lines that acquired drug resistance upon prolonged cultivation with irinotecan [49]. Since the reduction in doubling time was also observed in the control cell line BLUE-1 K10, the increased growth rate of both BLUE-1R10 and BLUE-1 K10 cannot be attributed to the effect of DZNep on the cells. Perhaps, some changes in genes responsible for cell cycle regulation and proliferation could have occurred during the longterm cultivation of the clone and its control. This is not surprising because, we already know the extent of genetic and transcriptional heterogeneity that occur in cell lines during evolution [50]. Besides, in other cell types 

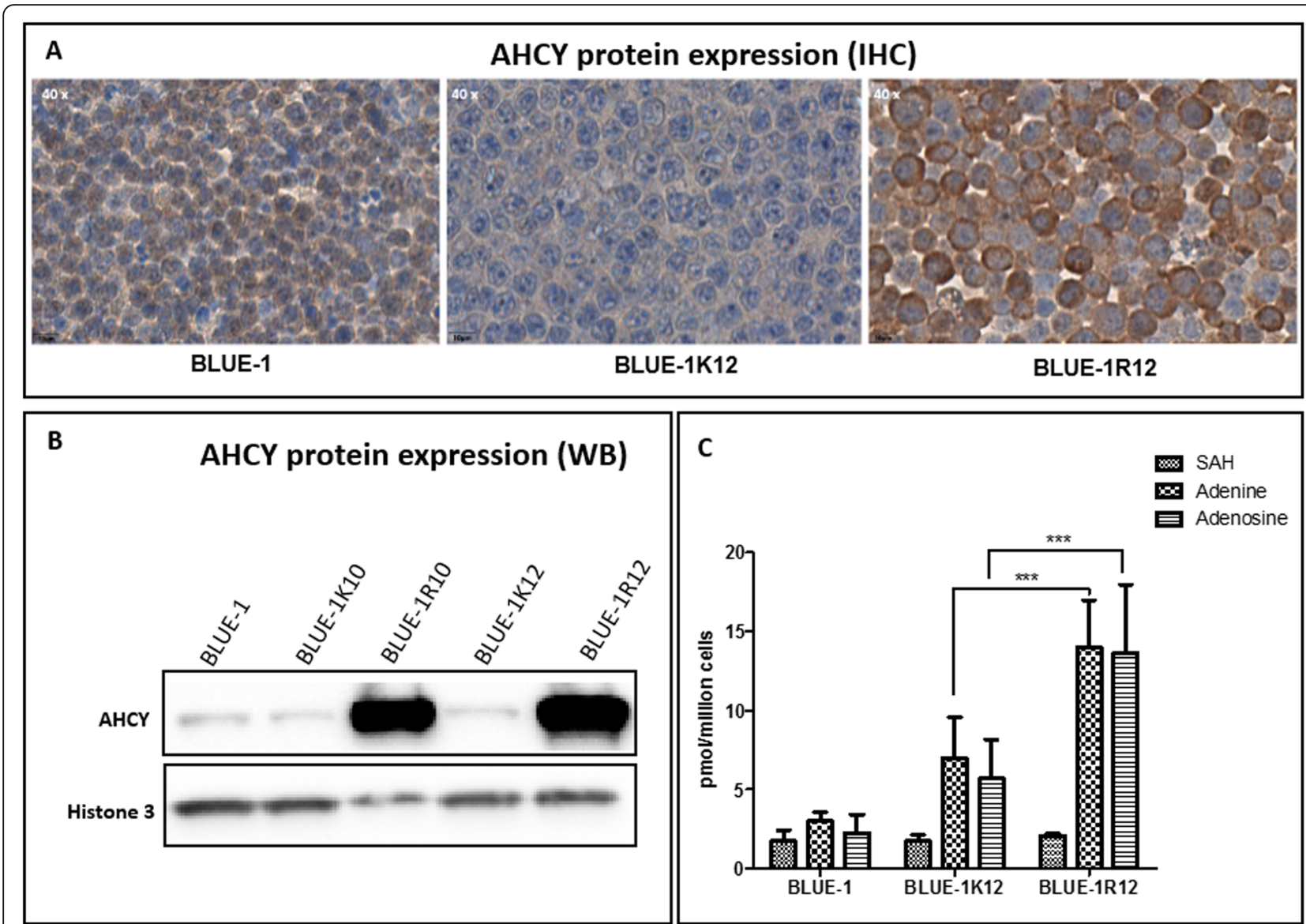

Fig. 4 Translational validation of AHCY gain in the resistant clone, and metabolomics analysis. a IHC results from BLUE-1, BLUE-1 K12 and BLUE1 R12 cell lines. Sections from FFPE cell line blocks were stained with anti-AHCY antibody. b Validation of AHCY overexpression at the protein level using Western blot. Whole cell protein lysates from the cell lines were analyzed using Western blot. Histone 3 was used as a loading control for the blot. The full-length blot is presented in Additional file 7: Fig. S6. FUSION-CAP Software was used for Western blot image analysis. c Quantification of S-adenosyl methionine, adenine and adenosine in BLUE-1, BLUE-1 K12 and BLUE-1R12. Values are shown as mean plus SD from six replicate measurements

such as human embryonic stem cells, the effect of prolonged cultivation on the proliferative capacity is evident as an increase in the proliferative capacity of these cells [51].

It was crucial to confirm the identity of the DZNepresistant clone to ensure that it certainly originated from the parent cell line BLUE-1. To achieve this, the STR profile and clonality of BLUE-1R10 was explored together with that of BLUE-1 K10 and BLUE-1. The identical STR profile and IGH chain gene rearrangement patterns indicate that both cell lines indeed originate from the same parent. Nevertheless, the differences observed between BLUE-1, BLUE-1 K10 and BLUE-1R10 upon genomic interrogation using the OncoScan CNV assay could suggest a divergent genomic evolution between BLUE-1 K10 and BLUE-1R10 cell lines in culture.

Since AHCY is a direct target of DZNep-mediated EZH2 inhibition, we focused on validating the identified $A H C Y$ gain in the resistant clone. Using the TaqMan
Copy Number Assay, which measures gene copy numbers by incorporating the TaqMan copy number assay with the TaqMan copy number reference assay in a single real-time PCR run, validation of $A H C Y$ amplification was achieved on the DNA level. The progressive increase in the $A H C Y$ copy number observed from BLUE-1R6 confirms that the gain in copy number is continual in the DZNep-resistant clone. It is noteworthy that at the point of DZNep withdrawal from the clone (from BLUE$1 R 9$ to BLUE-1R10), there was an almost two-fold increase in the $A H C Y$ copy number. This copy number gain doubled following continuous cultivation of the clone in a DZNep-free medium (from BLUE-1R10 to BLUE-1R11 and BLUE-1R12). This implies that the $A H C Y$ copy number gain, once initiated, does not require DZNep pressure to persist. Although this copy number event may reflect a sort of genomic compensation in the resistant clone due to prolonged AHCY inhibition, it is unlikely that this genomic aberration 
would decrease to baseline levels upon prolonged withdrawal of DZNep pressure.

Moreover, the FISH data which revealed that the $A H C Y$ amplification was more pronounced in BLUE1R12 in comparison to BLUE-1R10 further affirms the persistence of $A H C Y$ copy number gain. The chromosome 20 polysomy observed in the cells of each cell line may reflect the level of genomic instability usually occurring in cancer cells [52, 53]. The overexpression of AHCY on the transcriptional and protein level in the DZNep-resistant clone is consistent with the knowledge of drug resistance in cancer stemming from alterations in the drug target, particularly, alterations involving modified enzyme expression [46, 54]. Alterations in the apoptotic machinery did not appear to be involved in the resistance of the resistant clone to apoptosis based on its RNA expression profile.

The results of our metabolomics studies which revealed an analogous distribution for $\mathrm{SAH}$, adenine and adenosine in both BLUE-1 and BLUE-1 K12 signifies that following long-term culture of BLUE-1, there is no peculiar alteration of the balance exerted by these intermediates within the cell. The increase in adenine and adenosine levels of the resistant clone (BLUE-1R12) when compared to BLUE-1 K12 is in line with the increased expression of $\mathrm{AHCY}$, which catalyzes the hydrolysis of SAH to adenosine and L-homocysteine.

Previous works have linked copy number gains on chromosome 20q with the pathogenesis of tumors such as colon cancer [55], colorectal cancer [56], and cervical cancer [57], with significant gains of the $A H C Y$ gene among others. However, little is known about the role of $A H C Y$ copy number gain in driving B-cell lymphomas. For this reason, we analyzed the $A H C Y$ copy number of 12 primary lymphoma samples using the TaqMan CNV assay. The absence of $A H C Y$ copy number gain in the patient samples analyzed implies that it is unlikely that $A H C Y$ copy number gains play a driving role in B-cell lymphoma pathogenesis. In addition, the absence of AHCY copy number alterations from in silico studies signifies that alterations in $A H C Y$ are rare in primary Bcell lymphoma however, in solid tumors, the frequency of $A H C Y$ amplification can be as high as $20 \%[41,42]$.

\section{Conclusion}

Acquired drug resistance poses a great challenge in cancer therapy. It is important to recognize mechanisms of resistance for novel drugs about to enter clinical trials so that one can monitor for early signs of development of drug resistance. DZNep is a promising epigenetic drug that is in the pre-clinical phase of clinical approval, but has shown promising effects for selected cancer patients. We show that copy number gain of $A H C Y$ is one possible mechanism of acquired resistance to DZNep- mediated apoptosis and propose $\mathrm{AHCY}$ as a potential biomarker to stratify patients during the use of DZNep. Although AHCY alterations are rare in primary B-cell lymphomas, it may still be important to screen for modifications of this gene in patients prior to the initiation of EZH2 based therapy with DZNep. These findings might be valuable in predicting patients, who will benefit from EZH2 inhibition using DZNep once it progresses into clinical studies.

\section{Supplementary information}

Supplementary information accompanies this paper at https://doi.org/10. 1186/s12885-020-06937-8.

Additional file 1: Figure S1. Confirmation of the identical B-cell clonality of BLUE-1K10 and BLUE-1R10.

Additional file 2: Figure S2. Karyotypes of the cell lines BLUE-1, BLUE1 R10 and BLUE-1K10.

Additional file 3: Figure S3. OncoScan Copy number analysis in the of BLUE-1 cell lines.

Additional file 4: Figure S4. Analysis of $A H C Y$ copy number in primary lymphoma samples.

Additional file 5: Table S1. Comparative copy number analysis in the BLUE-1 cell lines as determined by Oncoscan assay.

Additional file 6: Figure S5. Full-length blot for Western blot image presented in Fig. 1.

Additional file 7: Figure S6. Full-length blot for Western blot image presented in Fig. 4.

\section{Abbreviations}

AHCY: S-adenosyl-L-homocysteine hydrolase; B2M: Beta 2 microglobulin; BAF: B-allele frequency; ChAS: Chromosome analysis suite; CN-LOH: Copy neutral loss of heterozygosity; CNR: Copy number ratio; CNV: Copy number variation; DZNep: 3-Deazaneplanocin A; DSMZ: German Collection of Microorganisms and Cell Cultures; EZH2: Enhancer of zeste homolog 2; FFPE: Formalin-fixed paraffin-embedded; FISH: Fluorescence in situ hybridization; H3K27me3: Lysine 27 trimethylation on histone 3; IGH: Immunoglobulin heavy chain; IHC: Immunohistochemistry; LOH: Loss of heterozygosity; RT-PCR: Reverse transcriptase polymerase chain reaction; SAH: S-adenosyl-L-homocysteine; SDHA: Succinate dehydrogenase; STR: Short tandem repeat; WES: Whole exome sequencing

\section{Acknowledgements}

We thank Anke Sommerfeld, Edda von der Wall, Erika Berg, Lisa Ellmann and the team of the tumor genetics group of the Institute of Human Genetics in Ulm for their technical support. We also appreciate Dr. Elena Myelona and Prof. Claudia Baldus for their constructive advice during this work.

\section{Authors' contributions}

CAA, KK, LD, MH took part in study conceptualization and design, CAA, KK, $E O, N T, K D, D L$ performed data curation. Data analysis and interpretation was carried out by CAA, KK, CM, CL, RW, KO, AS, DC, RS, LD and MH. CAA, PJO,

$\mathrm{DB}, \mathrm{RS}, \mathrm{DC}$ and $\mathrm{MH}$ acquired funding and resources. KK, RS, LD and MH supervised the project. CAA wrote the manuscript, and CAA, KK, CM, CL, RW, $\mathrm{KO}, \mathrm{KD}, \mathrm{AS}, \mathrm{PJO}, \mathrm{DB}, \mathrm{RS}, \mathrm{DC}$ and $\mathrm{MH}$ edited the manuscript. All authors read and approved the final manuscript.

\section{Funding}

This work, including personnel funding and acquisition of materials and reagents was supported by the Berlin School of Integrative Oncology (BSIO), Berlin, Germany. Infrastructural support by the KinderKrebsInitiative Buchholz Holm-Seppensen to the Institute of Human Genetics in Ulm is gratefully acknowledged. 


\section{Availability of data and materials}

The datasets supporting the conclusions of this article are included within the article and its additional files.

\section{Ethics approval and consent to participate}

Permission for the use of human primary samples was granted by the Institutional Review Board Charité - Berlin (EA4/104/11).

\section{Consent for publication}

Not applicable.

\section{Competing interests}

$\mathrm{MH}$ is a member of the editorial board (Associate Editor) of BMC cancer journal. All other authors declare that they have no competing interests.

\section{Author details}

${ }^{1}$ Department of Experimental Hematopathology, Institute of Pathology, Charité Medical University, Berlin, Charitéplatz 1, 10117 Berlin, Germany. ${ }^{2}$ Berlin School of Integrative Oncology, Charité - Medical University of Berlin, Berlin, Germany. ${ }^{3}$ Berlin Institute of Health, Charité Core Unit Bioinformatics, Berlin, Germany. Institute of Human Genetics, Ulm University and Ulm University Medical Center, Ulm, Germany. ${ }^{5}$ Institute of Functional Genomics, University of Regensburg, Regensburg, Germany. ${ }^{6}$ Department of Neuropathology, Charité, Medical University of Berlin, corporate member of Free University Berlin, Humboldt-University Berlin, and Berlin Institute of Health, Berlin, Germany. ${ }^{7}$ German Cancer Consortium (DKTK); Partner Site Berlin, German Cancer Research Center (DKFZ), Heidelberg, Germany.

\section{Received: 15 November 2019 Accepted: 7 May 2020}

\section{Published online: 14 May 2020}

\section{References}

1. Hu XT, He C. Recent progress in the study of methylated tumor suppressor genes in gastric cancer. Chin J Cancer. 2013;32(1):31-41.

2. Ghavifekr Fakhr M, Farshdousti Hagh M, Shanehbandi D, Baradaran B. DNA methylation pattern as important epigenetic criterion in cancer. Genet Res Int. 2013;2013:317569. https://doi.org/10.1155/2013/317569.

3. Martin C, Zhang Y. The diverse functions of histone lysine methylation. Nat Rev Mol Cell Bio. 2005;6(11):838-49.

4. Zhang $Y$, Reinberg D. Transcription regulation by histone methylation: interplay between different covalent modifications of the core histone tails. Genes Dev. 2001;15(18):2343-60.

5. Greer EL, Shi Y. Histone methylation: a dynamic mark in health, disease and inheritance. Nat Rev Genet. 2012;13(5):343-57.

6. Sadakierska-Chudy A, Filip M. A comprehensive view of the epigenetic landscape. Part II: histone post-translational modification, nucleosome level, and chromatin regulation by ncRNAs. Neurotox Res. 2015;27(2):172-97.

7. Grant PA. A tale of histone modifications. Genome Biol. 2001;2:4.

8. Barnes CE, English DM, Cowley SM. Acetylation \& co: an expanding repertoire of histone acylations regulates chromatin and transcription. Essays Biochem. 2019;63(1):97-107.

9. Alzrigat $M$, Jernberg-Wiklund H, Licht JD. Targeting EZH2 in Multiple Myeloma-Multifaceted Anti-Tumor Activity. Epigenomes. 2018;2(3):16.

10. Wang CX, Liu ZH, Woo CW, Li ZJ, Wang LF, Wei JS, et al. EZH2 mediates epigenetic silencing of neuroblastoma suppressor genes CASZ1, CLU, RUNX3, and NGFR. Cancer Res. 2012;72(1):315-24.

11. Yamaguchi H, Hung MC. Regulation and role of EZH2 in Cancer. Cancer Res Treat. 2014;46(3):209-22.

12. Gan L, Yang YN, Li Q, Feng Y, Liu TS, Guo WJ. Epigenetic regulation of cancer progression by EZH2: from biological insights to therapeutic potential. Biomark Res. 2018;6.

13. Kim KH, Roberts CWM. Targeting EZH2 in cancer. Nat Med. 2016;22(2):128-34

14. Marchesi I, Bagella L. Targeting enhancer of Zeste homolog 2 as a promising strategy for cancer treatment. World J Clin Oncol. 2016;7(2):135-48.

15. Tan J, Yang XJ, Zhuang L, Jiang $X$, Chen W, Lee PL, et al. Pharmacologic disruption of polycomb-repressive complex 2-mediated gene repression selectively induces apoptosis in cancer cells. Genes Dev. 2007;21(9):1050-63.

16. Fiskus W, Wang YC, Sreekumar A, Buckley KM, Shi HD, Jillella A, et al. Combined epigenetic therapy with the histone methyltransferase EZH2 inhibitor 3-deazaneplanocin a and the histone deacetylase inhibitor panobinostat against human AML cells. Blood. 2009;114(13):2733-43.
17. Zhou J, Bi C, Cheong LL, Mahara S, Liu SC, Tay KG, et al. The histone methyltransferase inhibitor, DZNep, up-regulates TXNIP, increases ROS production, and targets leukemia cells in AML. Blood. 2011;118(10):2830-9.

18. Fiskus W, Rao R, Balusu R, Ganguly S, Tao J, Sotomayor E, et al. Superior efficacy of a combined epigenetic therapy against human mantle cell lymphoma cells. Clin Cancer Res. 2012;18(22):6227-38.

19. Crea F, Hurt EM, Mathews LA, Cabarcas SM, Sun L, Marquez VE, et al. Pharmacologic disruption of Polycomb repressive complex 2 inhibits tumorigenicity and tumor progression in prostate cancer. Mol Cancer. 2011;10.

20. Akpa CA, Kleo K, Lenze D, Oker E, Dimitrova L, Hummel M. DZNepmediated apoptosis in B-cell lymphoma is independent of the lymphoma type, EZH2 mutation status and MYC, BCL2 or BCL6 translocations. PLoS One. 2019;14(8):e0220681.

21. Girard N, Bazille C, Lhuissier E, Benateau H, Llombart-Bosch A, Boumediene K, et al. 3-Deazaneplanocin A (DZNep), an Inhibitor of the Histone Methyltransferase EZH2, Induces Apoptosis and Reduces Cell Migration in Chondrosarcoma Cells. Plos One. 2014;9:5

22. Miranda TB, Cortez CC, Yoo CB, Liang GN, Abe M, Kelly TK, et al. DZNep is a global histone methylation inhibitor that reactivates developmental genes not silenced by DNA methylation. Mol Cancer Ther. 2009;8(6):1579-88.

23. Beluzic L, Grbesa I, Beluzic R, Park JH, Kong HK, Kopjar N, et al. Knock-down of AHCY and depletion of adenosine induces DNA damage and cell cycle arrest. Sci Rep. 2018;8(1):14012.

24. Park SJ, Kong HK, Kim YS, Lee YS, Park JH. Inhibition of Sadenosylhomocysteine hydrolase decreases cell mobility and cell proliferation through cell cycle arrest. Am J Cancer Res. 2015;5(7):2127-38.

25. Kim JH, Kim JH, Kim SC, Yi YS, Yang WS, Yang Y, et al. Adenosine dialdehyde suppresses MMP-9-mediated invasion of cancer cells by blocking the Ras/Raf-1/ERK/AP-1 signaling pathway. Biochem Pharmacol. 2013;86(9):1285-300

26. Li QH, Mao LH, Wang RL, Zhu LQ, Xue LX. Overexpression of Sadenosylhomocysteine hydrolase $(\mathrm{SAHH})$ in esophageal squamous cell carcinoma (ESCC) cell lines: effects on apoptosis, migration and adhesion of cells. Mol Biol Rep. 2014;41(4):2409-17.

27. Martinez-Climent JA, Alizadeh AA, Segraves R, Blesa D, Rubio-Moscardo F, Albertson DG, et al. Transformation of follicular lymphoma to diffuse large cell lymphoma is associated with a heterogeneous set of DNA copy number and gene expression alterations. Blood. 2003;101(8):3109-17.

28. Bookout AL, Mangelsdorf DJ. Quantitative real-time PCR protocol for analysis of nuclear receptor signaling pathways. Nucl Recept Signal. 2003;1: e012.

29. Li H. Aligning sequence reads, clone sequences and assembly contigs with BWA-MEM. arXivorg > q-bio > arXiv:13033997v2; 2013.

30. Faust GG, Hall IM. SAMBLASTER: fast duplicate marking and structural variant read extraction. Bioinformatics. 2014;30(17):2503-5.

31. Talevich E, Shain AH, Botton T, Bastian BC. CNVkit: genome-wide copy number detection and visualization from targeted DNA sequencing. PLoS Comput Biol. 2016;12(4):e1004873.

32. Wagener R, Lopez C, Kleinheinz K, Bausinger J, Aukema SM, Nagel I, et al. IG-MYC-positive neoplasms with precursor B-cell phenotype are molecularly distinct from Burkitt lymphomas. Blood. 2018;132:2280.

33. van Dongen JJM, Langerak AW, Bruggemann M, Evans PAS, Hummel M, Lavender FL, et al. Design and standardization of PCR primers and protocols for detection of clonal immunoglobulin and T-cell receptor gene recombinations in suspect lymphoproliferations: report of the BIOMED-2 concerted action BMH4-CT98-3936. Leukemia. 2003;17(12):2257-317.

34. Kleo K, Dimitrova L, Oker E, Tomaszewski N, Berg E, Taruttis F, et al. Identification of ADGRE5 as discriminating MYC target between Burkitt lymphoma and diffuse large B-cell lymphoma. BMC Cancer. 2019;19(1):322.

35. Horn H, Ziepert M, Wartenberg M, Staiger AM, Barth TFE, Bernd HW, et al. Different biological risk factors in young poor-prognosis and elderly patients with diffuse large B-cell lymphoma. Leukemia. 2015;29(7):1564-70.

36. Horn H, Ziepert M, Becher C, Barth TFE, Bernd HW, Feller AC, et al. MYC status in concert with BCL2 and BCL6 expression predicts outcome in diffuse large B-cell lymphoma. Blood. 2013;121(12):2253-63.

37. Schlegelberger B, Zwingers T, Harder L, Nowotny $H$, Siebert R, Vesely M, et al. Clinicopathogenetic significance of chromosomal abnormalities in patients with blastic peripheral B-cell lymphoma. Kiel-Wien-Lymphoma Study Group. Blood. 1999;94(9):3114-20.

38. Stevens AP, Dettmer K, Kirovski G, Samejima K, Hellerbrand C, Bosserhoff AK, et al. Quantification of intermediates of the methionine and polyamine 
metabolism by liquid chromatography-tandem mass spectrometry in cultured tumor cells and liver biopsies. J Chromatogr A. 2010;1217(19):3282-8.

39. Capper D, Stichel D, Sahm F, Jones DTW, Schrimpf D, Sill M, et al. Practical implementation of DNA methylation and copy-number-based CNS tumor diagnostics: the Heidelberg experience. Acta Neuropathol. 2018;136(2):181-210.

40. Hovestadt V, Zapatka M. Conumee: enhanced copy-number variation analysis using Illumina DNA methylation arrays. R packagehttp:// bioconductor.org/packages/conumee/.

41. Cerami E, Gao JJ, Dogrusoz U, Gross BE, Sumer SO, Aksoy BA, et al. The cBio Cancer genomics portal: an open platform for exploring multidimensional Cancer genomics data. Cancer Discov. 2012;2(5):401-4.

42. Gao JJ, Aksoy BA, Dogrusoz U, Dresdner G, Gross B, Sumer SO, et al. Integrative Analysis of Complex Cancer Genomics and Clinical Profiles Using the cBioPortal. Sci Signal. 2013;6(269):pl1.

43. Gottesman MM. Mechanisms of cancer drug resistance. Annu Rev Med. 2002;53:615-27.

44. Pan ST, Li ZL, He ZX, Qiu JX, Zhou SF. Molecular mechanisms for tumour resistance to chemotherapy. Clin Exp Pharmacol P. 2016;43(8):723-37.

45. Iglesias VS, Giuranno L, Dubois LJ, Theys J, Vooijs M. Drug resistance in nonsmall cell lung Cancer: a potential for NOTCH targeting? Front Oncol. 2018;8.

46. Mansoori B, Mohammadi A, Davudian S, Shirjang S, Baradaran B. The different mechanisms of Cancer drug resistance: a brief review. Adv Pharm Bull. 2017;7(3):339-48.

47. Cheng LL, Itahana Y, Lei ZD, Chia NY, Wu Y, Yu Y, et al. TP53 genomic status regulates sensitivity of gastric cancer cells to the histone methylation inhibitor 3-deazaneplanocin a (DZNep). Clin Cancer Res. 2012;18(15):4201-12.

48. Xie ZG, Bi CL, Cheong LL, Liu SC, Huang GF, Zhou JB, et al. Determinants of Sensitivity to DZNep Induced Apoptosis in Multiple Myeloma Cells. Plos One. 2011;6:6

49. Petitprez A, Poindessous V, Ouaret D, Regairaz M, Bastian G, Guerin E, et al, Acquired irinotecan resistance is accompanied by stable modifications of cell cycle dynamics independent of MSI status. Int J Oncol. 2013;42(5):1644-53.

50. Ben-David U, Siranosian B, Ha G, Tang H, Oren Y, Hinohara K, et al. Genetic and transcriptional evolution alters cancer cell line drug response. Nature. 2018:560(7718):325.

51. Bin Park Y, Kim YY, Oh SK, Chung SG, Ku SY, Kim SH, et al. Alterations of proliferative and differentiation potentials of human embryonic stem cells during long-term culture. Exp Mol Med. 2008;40(1):98-108.

52. Pikor L, Thu K, Vucic E, Lam W. The detection and implication of genome instability in cancer. Cancer Metast Rev. 2013;32(3-4):341-52.

53. Asatryan AD, Komarova NL. Evolution of genetic instability in heterogeneous tumors. J Theor Biol. 2016;396:1-12.

54. Housman G, Byler S, Heerboth S, Lapinska K, Longacre M, Snyder N, et al. Drug resistance in cancer: an overview. Cancers (Basel). 2014;6(3):1769-92.

55. Loo LWM, Tirikainen M, Cheng I, Lum-Jones A, Seifried A, Church JM, et al. Integrated analysis of genome-wide copy number alterations and gene expression in microsatellite stable, $\mathrm{CpG}$ island methylator phenotypenegative colon cancer. Gene Chromosome Canc. 2013;52(5):450-66.

56. Hassan NZA, Mokhtar NM, Sin TK, Rose IM, Sagap I, Harun R, et al. Integrated Analysis of Copy Number Variation and Genome-Wide Expression Profiling in Colorectal Cancer Tissues. Plos One. 2014;9(4):e92553.

57. Scotto L, Narayan G, Nandula S, Arias-Pulido H, Subramaniyam S, Schneider $\mathrm{A}$, et al. Identification of copy number gain and overexpressed genes on chromosome arm 20q by an integrative genomic approach in cervical cancer: potential role in progression. Gene Chromosome Canc. 2008;47(9): 755-65.

\section{Publisher's Note}

Springer Nature remains neutral with regard to jurisdictional claims in published maps and institutional affiliations.

Ready to submit your research? Choose BMC and benefit from:

- fast, convenient online submission

- thorough peer review by experienced researchers in your field

- rapid publication on acceptance

- support for research data, including large and complex data types

- gold Open Access which fosters wider collaboration and increased citations

- maximum visibility for your research: over $100 \mathrm{M}$ website views per year

At BMC, research is always in progress.

Learn more biomedcentral.com/submissions 